\title{
Implementation and axiomatization of discounted Shapley values
}

\author{
René van den Brink ${ }^{1}$ - Yukihiko Funaki
}

Received: 18 September 2014 / Accepted: 29 April 2015 / Published online: 5 June 2015

(C) The Author(s) 2015. This article is published with open access at Springerlink.com

\begin{abstract}
In this paper we introduce discounting in the bidding mechanism of PérezCastrillo and Wettstein (J Econ Theory 100:274-294, 2001) who implemented the Shapley value for cooperative transferable utility games. This modification of the mechanism yields the corresponding discounted Shapley value as the payoff distribution in every subgame perfect equilibrium. The class of discounted Shapley values contains the Shapley value and equal division solution as its extreme cases. Interestingly, we obtain axiomatizations of each solution in this class by generalizing the null player property (of the Shapley value) and nullifying player property (of the equal division solution) to the so-called $\delta$-reducing player property.
\end{abstract}

Mathematics Subject Classification $\quad 91 \mathrm{~A} 10 \cdot 91 \mathrm{~A} 12$

JEL-Codes C71 · C72

\section{Introduction}

One of the most well-known solutions for cooperative transferable utility games is the Shapley value introduced and axiomatized in Shapley (1953). Pérez-Castrillo and Wettstein (2001) provided a strategic (noncooperative) foundation by introducing a

René van den Brink

jrbrink@feweb.vu.nl

Yukihiko Funaki

funaki@waseda.jp

1 Department of Econometrics and Tinbergen Institute, VU University, De Boelelaan 1105, 1081 HV Amsterdam, The Netherlands

2 Faculty of Political Science and Economics, Waseda University, 1-6-1 Nishi-Waseda, Shinjuku-Ku, Tokyo 169-8050, Japan 
bidding mechanism that implements the Shapley value payoffs as unique subgame perfect equilibrium payoffs.

In this paper we explore the effect of introducing discounting in this bidding mechanism. Assuming that after each rejection of the proposal the amount available to be distributed among the remaining players in the next round is discounted by a parameter $\delta \in[0,1]$, we show that this modification of the mechanism of Pérez-Castrillo and Wettstein (2001) yields the corresponding $\delta$-discounted Shapley value as the payoff distribution in every subgame perfect equilibrium of this game. ${ }^{1}$ These solutions are introduced by Joosten (1996) who showed that all of them satisfy the famous Hart and Mas-Colell reduced game consistency [(see Hart and Mas-Colell $(1988,1989)]$. This class of solutions contains the Shapley value (for $\delta=1$ ) and equal division solution (for $\delta=0$ ) as extreme cases. Therefore, the solutions characterized by this bidding mechanism make a trade-off between marginalism and egalitarianism, such that the higher (lower) the discount factor the more weight is put on marginalism (egalitarianism).

Following Nash (1953), besides this implementation we provide an axiomatization of the discounted Shapley values. Most axiomatic characterizations of the Shapley value use some axiom related to null players (i.e. players whose marginal contribution to any coalition is zero). An axiomatic comparison of the Shapley value and the equal division solution is made by van den Brink (2007) who considers several axiomatizations of the Shapley value and shows that replacing an axiom concerning null players by a similar axiom concerning nullifying players (i.e. players whose presence in a coalition implies the coalition earns zero worth) characterizes the equal division solution. For example, in the original axiomatization of the Shapley value by efficiency, the null player property, symmetry and additivity, replacing the null player property (which states that null players receive a zero payoff) by the nullifying player property (which states that nullifying players receive a zero payoff) characterizes the equal division solution. In the underlying paper we generalize both axioms by introducing a $\delta$-reducing player in a game as a player such that any coalition containing this player earns a fraction $\delta \in[0,1]$ of the worth of that coalition without this player. Then the $\delta$-reducing player property states that such a player receives a zero payoff. Clearly, $\delta=1$ implies that player $i$ 's marginal contributions are all zero, and thus yields the null player property of the Shapley value. On the other hand, $\delta=0$ implies that the worth of any coalition containing player $i$ is zero, and thus this yields the nullifying player property. It turns out that replacing the null or nullifying player property (in the axiomatizations of the Shapley value, respectively, equal division solution) by this $\delta$-reducing player property characterizes the corresponding $\delta$-discounted Shapley value. Besides the original axiomatization of Shapley (1953) we also adapt the characterizations of Young (1985) and Chun (1991).

The paper is organized as follows. Section 2 contains preliminaries on TU-games and solutions. In Sect. 3 we provide a strategic implementation of the discounted Shapley values. In Sect. 4 we provide axiomatic characterizations. Finally, Sect. 5 contains concluding remarks.

\footnotetext{
1 So, the discounting in our mechanism is similar to that in Rubinstein (1982)'s 'fixed discounting factor' bargaining model but with the same discount factor for all players.
} 


\section{Preliminaries}

A situation in which a finite set of players can obtain certain payoffs by cooperation can be described by a cooperative game with transferable utility, or simply a TUgame, being a pair $(N, v)$, where $N \subset \mathbb{N}$ is a finite set of players and $v: 2^{N} \rightarrow \mathbb{R}$ is a characteristic function on $N$ such that $v(\varnothing)=0$. For any coalition $S \subseteq N, v(S)$ is called the worth of coalition $S$. This is the transferable utility that the members of coalition $S$ can obtain by agreeing to cooperate. We denote the class of all TU-games by $\mathcal{G}$. In the sequel we denote $n=|N|$ for the number of players in $N$. For generic coalitions $S \subseteq N$ we denote $s=|S|$.

A payoff vector of game $(N, v)$ is an $n$-dimensional real vector $x \in \mathbb{R}^{N}$ which represents a distribution of the payoffs that can be earned by cooperation over the individual players. A (point-valued) solution for TU-games is a function $\psi$ which assigns a payoff vector $\psi(N, v) \in \mathbb{R}^{N}$ to every TU-game $(N, v) \in \mathcal{G}$ such that $\psi_{i}(\{i\}, v)=v(\{i\})$ if $N=\{i\}$. Two well-known solutions are the Shapley value and the equal division solution.

The increase in worth when player $i \in N$ joins coalition $S \subseteq N \backslash\{i\}$ is called the marginal contribution of player $i$ to coalition $S$ and is denoted by

$$
m_{i}^{S}(v)=v(S \cup\{i\})-v(S) .
$$

Suppose that the 'grand coalition' $N$ forms in a way such that the players enter the coalition one by one. Such an order of entrance can be represented by a permutation $\pi: N \rightarrow N$ of the players. We denote the collection of all permutations on $N$ by $\Pi(N)$. For every $\pi \in \Pi(N)$ we denote by $P(\pi, i)=\{j \in N \mid \pi(j)<\pi(i)\}$ the set of players that enter before player $i$ in the order $\pi$. The Shapley value (Shapley 1953 ) is the solution $S h: \mathcal{G}^{N} \rightarrow \mathbb{R}^{N}$ that assigns to every player its expected marginal contribution to the coalition of players that enter before him, given that every order of entrance $\pi$ has equal probability $\frac{1}{n !}$ to occur, i.e.

$S h_{i}(v)=\frac{1}{n !} \sum_{\pi \in \Pi(N)} m_{i}^{P(\pi, i)}(v)=\sum_{S \subseteq N \backslash\{i\}} \frac{(n-|S|-1) !(|S|) !}{n !} \cdot m_{i}^{S}(v)$ for all $i \in N$

The equal division solution is the solution that distributes the worth $v(N)$ of the 'grand coalition' equally among all players, and thus assigns to every TU-game $(N, v)$ the payoff vector

$$
E D_{i}(N, v)=\frac{v(N)}{n} \text { for all } i \in N
$$

Following a similar procedure as above, i.e. the 'grand coalition' $N$ forms by the players entering the coalition one by one, it follows from a more general result in Malawski (2013) that allocating the marginal contribution of a player $i$ equally among its predecessors in $P(\pi, i)$, the average payoff over all permutations yields the equal division solution. 
Joosten (1996) introduces two classes of solutions that contain the Shapley value and equal division solution as extreme cases. One is the class of egalitarian Shapley values being convex combinations of the Shapley value and equal division solution. The other is the class of solutions that were later called $\delta$-discounted Shapley values by Driessen and Radzik (2002). For $\delta \in[0,1]$, the corresponding $\delta$-discounted Shapley value $S h^{\delta}$ is given by

$$
S h_{i}^{\delta}(N, v)=\sum_{S \subseteq N \backslash\{i\}} \frac{s !(n-s-1) !}{n !} \delta^{n-s-1}(v(S \cup\{i\})-\delta \cdot v(S)) \text { for all } i \in N .
$$

Clearly, taking $\delta=1$ yields the Shapley value, while taking $\delta=0$ yields the equal division solution.

Finally, for $\delta \in[0,1]$, a TU-game is called $\delta$-monotonic if $v(S) \geq \delta v(S \backslash\{i\})+$ $v(\{i\})$ for all $S \subseteq N$ and all $i \in S$. Taking $\delta=1$ yields the well-known zeromonotonicity. Note that for nonnegative games (i.e. $v(S) \geq 0$ for all $S \subseteq N$ ), for any pair of discount factors $\delta, \delta^{\prime} \in[0,1]$ with $\delta<\delta^{\prime}$, a nonnegative game is $\delta$-monotonic if it is $\delta^{\prime}$-monotonic. So, $\delta$-monotonicity is weaker than zero-monotonicity for every $\delta \in(0,1]$, and thus also weaker than superadditivity. ${ }^{2}$

\section{Implementation}

In the literature various implementations of the Shapley value can be found, see e.g. Gul (1989), Hart and Mas-Colell (1996) and Pérez-Castrillo and Wettstein (2001). A cooperative game solution is (strategically) implemented if for every cooperative game one defines an associated strategic or extensive form game (a mechanism) such that applying some strategic solution, for example the subgame perfect equilibrium, always yields payoff outcomes according to this cooperative solution. In this paper we modify the bidding mechanism of Pérez-Castrillo and Wettstein (2001) by introducing discounting of the worths to be allocated, each time a proposal is rejected and the remaining players move to the next round. The bidding mechanism of Pérez-Castrillo and Wettstein (2001) proceeds in rounds, where each round consists of four stages. In the first stage of round 1, all players make bids to each other and the player with the highest net bid (being the difference between the sum of all bids made to the other players and the sum of all bids received from the other players) is chosen to be the proposer in stage 2 (where ties are broken by assigning each of the players with highest net bid to be the proposer with equal probability). We denote this winner by $\alpha_{1}$. The 'winner' $\alpha_{1}$ of this bidding pays the other players the bids that it offered. In the second stage the proposer $\alpha_{1}$ makes a proposal (i.e. proposes a payoff) to every other player. In stage 3 the other players, sequentially, accept or reject the proposal. The proposal is accepted if all other players accept, and is rejected if at least one other player rejects it. In stage 4 , if the proposal is accepted the players are paid the proposed payoffs (additional to the bids made by the proposer in stage 1). If the proposal is rejected then

\footnotetext{
2 A TU-game $(N, v)$ is superadditive if $v(S \cup T)+v(S \cap T) \geq v(S)+v(T)$ for all $S, T \subseteq N$ with $S \cap T=\emptyset$.
} 
the proposer $\alpha_{1}$ leaves the game and receives its own singleton worth (from which it has to pay the bids made in stage 1) while the other players go to the next round to bid and bargain over their worth $v\left(N \backslash\left\{\alpha_{1}\right\}\right)$.

The second round has the same four stages as the first round (but with $n-1$ players). The game proceeds untill at some round all remaining players accept the offer made by the proposer of that round or, after a sequence of rejections, round $n$ is reached in which there is only one player who just gets its singleton worth (plus all the net bids it received in all the first bidding stages of all rounds). Pérez-Castrillo and Wettstein (2001) showed that, if the cooperative TU-game $(N, v)$ is zero-monotonic then the payoffs in any subgame perfect equilibrium of this extensive form game are equal to the Shapley value of $(N, v)$.

In this section we modify this bidding mechanism by discounting the worths to be allocated each time a proposal is rejected and the players move to a next round. So, after the proposal of player $\alpha_{1}$ in the first round is rejected, the remaining players continue to bargain over $\delta v\left(N \backslash\left\{\alpha_{1}\right\}\right)$ instead of $v\left(N \backslash\left\{\alpha_{1}\right\}\right)$. We assume that the same discount factor $\delta \in[0,1]$ is applied in every round. So, in round $t \in\{1, \ldots, n\}$ the remaining players bargain over $\delta^{t-1}$ of their worth.

To formally describe the bidding mechanism, let $N_{t}$ be the player set of the game with which each round $t \in\{1, \ldots, n\}$ will start, so $N_{1}=N$. For $T \subset N$ the restricted game $\left(T, v_{T}\right) \in \mathcal{G}$ is given by $v_{T}(S)=v(S)$ for all $S \subseteq T$.

\section{The bidding mechanism}

Round $t, t \in\{1, \ldots, n-1\}$ :

Stage 1: Each player $i \in N_{t}$ makes bids $b_{j}^{i} \in \mathbb{R}$ for every $j \neq i$. For each $i \in N_{t}$, let $B^{i}=\sum_{j \in N_{t} \backslash\{i\}}\left(b_{j}^{i}-b_{i}^{j}\right)$, be the net bid of player $i$. Let $\alpha_{t}$ be the player with the highest net bid of round $t$. (In case of a non-unique maximizer we choose any of these maximal bidders to be the 'winner' with equal probability.) Once $\alpha_{t}$ has been chosen, player $\alpha_{t}$ pays every other player $j \in N_{t} \backslash\left\{\alpha_{t}\right\}$, its offered bid $b_{j}^{\alpha_{t}}$. Player $\alpha_{t}$ becomes the proposer in the next stage. Go to Stage 2.

Stage 2: Player $\alpha_{t}$ proposes an offer $y_{j}^{\alpha_{t}} \in \mathbb{R}$ to every player $j \in N_{t} \backslash\left\{\alpha_{t}\right\}$. (This offer is additional to the bids paid at stage 1.) Go to Stage 3.

Stage 3: The players other than $\alpha_{t}$, sequentially, either accept or reject the offer. If at least one player rejects it, then the offer is rejected. Otherwise, the offer is accepted. Go to Stage 4.

Stage $4^{3}$ : If the offer is accepted, then each player $j \in N_{t} \backslash\left\{\alpha_{t}\right\}$ receives $y_{j}^{\alpha_{t}}$ and player $\alpha_{t}$ obtains the remainder $\delta^{t-1} v\left(N_{t}\right)-\sum_{j \in N_{t} \backslash\left\{\alpha_{t}\right\}} y_{j}^{\alpha_{t}}$ of the discounted payoff at this stage in round $t$. Hence, in this case the final payoff to player $j \in N_{t} \backslash\left\{\alpha_{t}\right\}$ is $y_{j}^{\alpha_{t}}+b_{j}^{\alpha_{t}}+\sum_{k=1}^{t-1} b_{j}^{\alpha_{k}}$, while player $\alpha_{t}$ receives $\delta^{t-1} v\left(N_{t}\right)-$ $\sum_{j \in N_{t} \backslash\left\{\alpha_{t}\right\}}\left(y_{j}^{\alpha_{t}}+b_{j}^{\alpha_{t}}\right)+\sum_{k=1}^{t-1} b_{\alpha_{t}}^{\alpha_{k}}$. Stop.

If the offer is rejected then player $\alpha_{t}$ leaves the game and obtains its discounted stand-alone payoff $\delta^{t-1} v\left(\left\{\alpha_{t}\right\}\right)$, while the players in $N_{t} \backslash\left\{\alpha_{t}\right\}$

\footnotetext{
3 Note that in round $t$ we have $\left|N_{t}\right|=n-t+1$. Besides, if player $\alpha_{t}$ is a proposer in round $t$ then all the proposers from earlier rounds, $\alpha_{1}, \alpha_{2}, \ldots, \alpha_{t-1}$ are different from player $\alpha_{t}$.
} 
proceed to round $t+1$ to bargain over $\delta^{t} v\left(N_{t} \backslash\left\{\alpha_{t}\right\}\right)$. So, the final payoff for player $\alpha_{t}$ is $\delta^{t-1} v\left(\left\{\alpha_{t}\right\}\right)+\sum_{k=1}^{t-1} b_{\alpha_{t}}^{\alpha_{k}}$.

Round $n: N_{n}=N_{n-1} \backslash\left\{\alpha_{n-1}\right\}$. Since $N_{n}$ is a singleton coalition it is a one-player (sub)game in this round. The game immediately stops such that player $i \in N_{n}$ gets its discounted stand-alone payoff $\delta^{n-1} v\left(N_{n}\right)$. Its final payoff thus is $\delta^{n-1} v\left(N_{n}\right)+$ $\sum_{k=1}^{n-1} b_{i}^{\alpha_{k}}$.

As mentioned above, Pérez-Castrillo and Wettstein (2001) showed that for $\delta=1$ this bidding mechanism implements the Shapley value for zero-monotonic games. Next we evaluate the subgame perfect equilibrium outcomes of this game for any discount factor. It turns out that for any $\delta \in[0,1]$ the above mechanism implements the corresponding $\delta$-discounted Shapley value in SPE. ${ }^{4}$

Theorem 3.1 Let $\delta \in[0,1]$, and let $(N, v) \in \mathcal{G}$ be a $\delta$-monotonic game.

Then the outcome in any subgame perfect equilibrium of the bidding mechanism is equal to $\operatorname{Sh}^{\delta}(N, v)$.

Proof Here we only give a brief intuition behind the proof. A more detailed proof can be found in Sect. 5. Take $\delta \in[0,1]$ and $(N, v) \in \mathcal{G}$. It has been shown by Driessen and Radzik (2002) that, for an $n$-player game $(N, v)$, the solutions $S h^{\delta}$ can be obtained by applying the Shapley value to the modified game $\left(N, v^{\delta}\right)$ given by $v^{\delta}(S)=\delta^{n-s} v(S)$, $S \subseteq N$, i.e. for every $\delta \in[0,1]$ it holds that

$$
\operatorname{Sh}^{\delta}(N, v)=\operatorname{Sh}\left(N, v^{\delta}\right)
$$

But then, for every $\delta \in[0,1]$ and $(N, v) \in \mathcal{G}$, applying the mechanism of PérezCastrillo and Wettstein (2001) to the game $\left(N, v^{\delta}\right)$ implements $\operatorname{Sh}^{\delta}(N, v)=\operatorname{Sh}\left(N, v^{\delta}\right)$ in the sense that the outcome in any subgame perfect equilibrium of this mechanism is equal to $S h^{\delta}(N, v)$. Let us refer to the mechanism of Pérez-Castrillo and Wettstein (2001) applied to $\left(N, v^{\delta}\right)$ as the PCW-2 mechanism. Note that this is not exactly the same as the mechanism described above since in any round $t$, if the proposal is rejected then the proposer $\alpha_{t}$ leaves the game with payoff $v^{\delta}\left(\left\{\alpha_{t}\right\}\right)=\delta^{n-1} v\left(\left\{\alpha_{t}\right\}\right)$ according to the PCW-2 mechanism, while the proposer leaves with payoff $\delta^{t-1} v\left(\left\{\alpha_{t}\right\}\right)$ in the mechanism above. ${ }^{5}$ However, the offers to be accepted by the other players in round $t$ are determined by the SPE strategies in the subgame that starts after rejection of the proposal, and it is known that the proposer in SPE of the PCW-2 mechanism makes a proposal that will be accepted. Although the payoff for the proposer after rejection in our mechanism $\left(\delta^{t-1} v\left(\alpha_{t}\right)\right)$ is at least as much as in the PCW-2 mechanism $\left(\delta^{n-1} v\left(\alpha_{t}\right)\right)$, with equality if $t=n$ or $\delta \in\{0,1\}$, it can be shown that this does not affect the subgame perfect equilibrium outcomes. As mentioned, a more detailed proof can be found in Sect. 5 .

\footnotetext{
4 We remark that under the alternative condition $v(S) \geq \delta(v(S \backslash\{i\})+v(\{i\}))$ in stage 4 of the bidding mechanism we could allow proposer $\alpha_{t}$ in round $t$ to obtain $\delta^{t} v\left(\alpha_{t}\right)$ if its proposal is rejected.

5 Note that the worth to be allocated in round $t$ in the PCW-2 mechanism is $\delta^{n-\left|N_{t}\right|} v\left(N_{t}\right)=$ $\delta^{n-(n-t+1)} v\left(N_{t}\right)=\delta^{t-1} v\left(N_{t}\right)$, and thus is the same as the payoff to be allocated in our mechanism above.
} 
Note that, since for nonnegative games $\delta$-monotonicity, with $\delta \in(0,1]$, is weaker than zero-monotonicity and thus also weaker than superadditivity, the implementation also holds if we just require the game to be nonnegative and zero-monotonic (or superadditive).

Theorem 3.1 gives a characterization of each $\delta$-discounted Shapley value. The difference is a discount factor $\delta$ in the process of the bidding mechanism which reflects the higher cost of longer bargaining, similar to Rubinstein (1982)'s bargaining process.

Interestingly, Joosten (1996) showed that all these solutions satisfy Hart and MasColell (HM) consistency, ${ }^{6}$ see Hart and Mas-Colell (1988, 1989), irrespective of the discount factor $\delta$. Even stronger, he showed that for any $\delta \in[0,1]$ the corresponding $\delta$-discounted Shapley value is characterized by HM-consistency and $\delta$-standardness for two player games. ${ }^{7}$ So, as a corollary from Theorem 3.1 we obtain the following.

Corollary 3.2 Let $\delta \in[0,1]$. The solution that assigns to every $(N, v) \in \mathcal{G}$ the $\operatorname{SPE}$ payoffs of the corresponding bidding mechanism satisfies HM-consistency.

In the bidding mechanism above we presented the parameter $\delta$ as a discount factor which determines the discounting of the available worth going from one round of negotiation to the next after a rejection of the proposal. Alternatively, the parameter $\delta$ can be interpreted as a probability of breakdown of the negotiations after a rejection. In van den Brink et al. (2013) the mechanism of Pérez-Castrillo and Wettstein (2001) is adapted by allowing this possibility of breakdown of the negotiations only after rejection of the proposal in the first round of the negotiations. After that the mechanism is the same as that of Pérez-Castrillo and Wettstein (2001) so there is no possibility of breakdown anymore. This kind of breakdown may occur, for example, when the players do not know each other and speak different languages. Then it might be that after rejection in the first round they decide that they cannot communicate and bargain with each other. However, if they reject the proposal but agree to continue negotiations, then then they will bargain untill they reach an agreement or only one player is left. In van den Brink et al. (2013) it is shown that allowing breakdown of the negotiations only in the first round implements the so-called egalitarian Shapley values, also introduced by Joosten (1996), being convex combinations of the Shapley value and equal division solution. ${ }^{8}$

\section{Axiomatization}

Next we turn our attention to an axiomatization of the discounted Shapley values. In the literature several axiomatizations of the Shapley value can be found. We first

\footnotetext{
${ }^{6}$ Solution $\psi$ satisfies $H M$-consistency if $\psi_{i}(N, v)=\psi_{i}\left(T, \bar{v}_{T}^{\psi}\right)$ for every $(N, v) \in \mathcal{G}, T \subseteq N$, and $i \in T$, where the reduced game $\left(T, \bar{v}_{T}^{\psi}\right)$ is given by $\bar{v}_{T}^{\psi}(S)=v\left(S \cup T^{c}\right)-\sum_{j \in T^{c}} \psi_{j}\left(S \cup T^{c}, v_{S \cup T^{c}}\right)$ for all $S \subseteq T$ with $T^{c}=N \backslash T$.

${ }^{7}$ For $\delta \in[0,1], \delta$-standardness for two player games means that in a two player game each player gets a fraction $\delta$ of its singleton worth, and the remaining surplus is distributed equally among the two players.

${ }^{8}$ For every $\alpha \in[0,1]$, the $\alpha$-egalitarian Shapley value $\varphi^{\alpha}$ is the solution given by $\varphi^{\alpha}(N, v)=\alpha \operatorname{Sh}(N, v)+$ $(1-\alpha) E D(N, v)$.
} 
consider the 'classical' axiomatization of Shapley (1953) and the modification for the equal division solution given in van den Brink (2007). Both solutions satisfy the well-known axioms of efficiency, symmetry and linearity. A solution $\psi$

- is efficient ${ }^{9}$ if $\sum_{i \in N} \psi_{i}(N, v)=v(N)$ for all $(N, v) \in \mathcal{G}$;

- is symmetric if $\psi_{i}(N, v)=\psi_{j}(N, v)$ for all $(N, v) \in \mathcal{G}$ and $i, j \in N$ such that $v(S \cup\{i\})=v(S \cup\{j\})$ for all $S \subseteq N \backslash\{i, j\}$;

- is linear if $\psi(N, \beta v+\gamma w)=\beta \psi(N, v)+\gamma \psi(N, w)$ for all $(N, v),(N, w) \in \mathcal{G}$ and $\beta, \gamma \in \mathbb{R}$, where $\beta v+\gamma w$ is given by $(\beta v+\gamma w)(S)=\beta v(S)+\gamma w(S)$ for all $S \subseteq N$.

Shapley (1953) characterized the Shapley value by efficieny, symmetry, linearity and the null player property. ${ }^{10}$ Player $i \in N$ is a null player in game $(N, v)$ if $m_{i}^{S}(v)=0$ for all $S \subseteq N$. A solution $\psi$

- satisfies the null player property if $\psi_{i}(N, v)=0$ whenever $i$ is a null player in $(N, v)$.

In van den Brink (2007) it is shown that replacing the null player property by the nullifying player property characterizes the equal division solution. Player $i \in N$ is a nullifying player in game $(N, v)$ if $v(S)=0$ for all $S \subseteq N$ with $i \in S$. A solution $\psi$

- satisfies the nullifying player property ${ }^{11}$ if $\psi_{i}(N, v)=0$ whenever $i$ is a nullifying player in $(N, v)$.

We generalize the null- and nullifying player property as follows. For $\delta \in[0,1]$ we call player $i \in N$ a $\delta$-reducing player in game $(N, v)$ if $v(S \cup\{i\})=\delta v(S)$ for all $S \subseteq N \backslash\{i\}$. So, when player $i$ enters any coalition then the worth of this coalition is a fraction $\delta$ of the worth of the coalition without player $i$. Clearly, if $\delta=1$ this implies that the worth of no coalition changes when $i$ enters, i.e. a 1-reducing player is a null player. When $\delta=0$ then the worth of any coalition not containing $i$ becomes zero when $i$ enters, i.e. a 0 -reducing player is a nullifying player. Consequently the following property generalizes the null- as well as the nullifying player property.

- For $\delta \in[0,1]$, solution $\psi$ satisfies the $\delta$-reducing player property if $\psi_{i}(N, v)=0$ whenever $i$ is a $\delta$-reducing player in $(N, v)$.

Next, we show that this axiom together with efficiency, symmetry and linearity characterizes the corresponding $\delta$-discounted Shapley value for any $\delta \in[0,1]$. This follows similar as axiomatizations of the Shapley value and equal division solution, but using a different basis for TU-games. Consider a fixed player set $N$. For every $T \subseteq N, T \neq \emptyset$, and $\delta \in[0,1]$ we define the game $\left(N, d_{T}^{\delta}\right) \in \mathcal{G}$ by

$$
d_{T}^{\delta}(S)= \begin{cases}\delta^{s-t} & \text { if } T \subseteq S \\ 0 & \text { otherwise }\end{cases}
$$

\footnotetext{
9 Efficient solutions are often called values.

10 This axiomatization is more often presented in this way although Shapley (1953) combines efficiency and the null player property into a carrier axiom.

11 Deegan and Packel (1979) refer to nullifying players as zero players and use this property to characterize their (non-efficient) Deegan-Packel value.
} 
To illustrate these games, consider coalition $T=\{1\}$ of the player set $N=\{1,2,3\}$. Then

$$
d_{T}^{\delta}(S)= \begin{cases}1 & \text { if } S=\{1\} \\ \delta & \text { if } S \in\{\{1,2\},\{1,3\}\} \\ \delta^{2} & \text { if } S=\{1,2,3\} \\ 0 & \text { otherwise }\end{cases}
$$

Note that taking $\delta=1$, the game $d_{T}^{\delta}$ is the unanimity game of coalition $T \subseteq N$, $T \neq \emptyset$, i.e.

$$
d_{T}^{1}(S)= \begin{cases}1 & \text { if } T \subseteq S \\ 0 & \text { otherwise }\end{cases}
$$

It is well-known that every game can be expressed as a unique linear combination of unanimity games by $v=\sum_{\substack{T \subseteq N \\ T \neq \emptyset}} \Delta_{v}(T) d_{T}^{1}$ with $\Delta_{v}(T)=\sum_{S \subseteq T}(-1)^{|T|-|S|} v(S)$ the Harsanyi dividends, see Harsanyi (1959). This fact is used by Shapley (1953) to show that the Shapley value is the unique solution satisfying efficiency, symmetry, linearity and the null player property (where all players in $N \backslash T$ are null players in $d_{T}^{1}$.). On the other extreme, taking $\delta=0$ the game $d_{T}^{\delta}$ is the standard game of coalition $T \subseteq N$, $T \neq \emptyset$, i.e.

$$
d_{T}^{0}(S)= \begin{cases}1 & \text { if } T=S \\ 0 & \text { otherwise }\end{cases}
$$

Also, every game can be expressed as a unique linear combination of standard games as $v=\sum_{\substack{T \subseteq N \\ T \neq \emptyset}} v(T) d_{T}^{0}$. This fact is used by van den Brink (2007) to show that the equal division solution is the unique solution satisfying efficiency, symmetry, linearity and the nullifying player property (where all players in $N \backslash T$ are nullifying players in $d_{T}^{0}$ ).

Next, we first show that also the games $d_{T}^{\delta}$ form a basis for the class of TU-games.

Lemma 4.1 For every $(N, v) \in \mathcal{G}$ and $\delta \in[0,1]$ there exist unique numbers $D_{v}^{\delta}(T) \in$ $\mathrm{R}$ such that $v=\sum_{\substack{T \subseteq N \\ T \neq \emptyset}} D_{v}^{\delta}(T) d_{T}^{\delta}$.

Proof Consider a game $(N, v) \in \mathcal{G}$ and $\delta \in[0,1]$. Obviously, if $T=\{i\}$ then $D_{v}^{\delta}(\{i\})=v(\{i\})$ is uniquely determined since $d_{\{i\}}^{\delta}$ is the only game $d_{T}^{\delta}$ for which $d_{T}^{\delta}(\{i\}) \neq 0$. Proceeding by induction, suppose that the numbers $D_{v}^{\delta}\left(T^{\prime}\right)$ have been uniquely determined for any $T^{\prime} \subset N$ with $\left|T^{\prime}\right|<|T|$. Since the worth of any $S \subseteq N$ can be written as $v(S)=\sum_{H \subseteq N} D_{v}^{\delta}(H) d_{H}^{\delta}(S)=\sum_{H \subseteq S} D_{v}^{\delta}(H) d_{H}^{\delta}(S)=$ $D_{v}^{\delta}(S) d_{S}^{\delta}(S)+\sum_{H \subset S} D_{v}^{\delta}(H) d_{H}^{\delta}(S)$, it follows with the induction hypothesis and $d_{T}^{\delta}(T)=1$, that $D_{v}^{\delta}(T)=\frac{1}{d_{T}^{\delta}(T)}\left(v(T)-\sum_{H \subset T} D_{v}^{\delta}(H) d_{H}^{\delta}(T)\right)$ is uniquely determined. 
Next, we can state our second main result, axiomatizing the $\delta$-discounted Shapley values. $^{12}$

Theorem 4.2 Take $\delta \in[0,1]$. Solution $\psi$ satisfies efficiency, symmetry, linearity and the $\delta$-reducing player property if and only if $\psi=S h^{\delta}$.

Proof It is straightforward to verify that $S h^{\delta}$ satisfies these axioms. To show uniqueness ${ }^{13}$ suppose that solution $\psi$ satisfies the four axioms, and take $T \subset N, T \neq \emptyset$. We first show that every player $i \in N \backslash T$ is a $\delta$-reducing player in $\left(N, d_{T}^{\delta}\right)$. Consider $S \subseteq N \backslash\{i\}$. We distinguish two cases.

(i) Suppose that $T \nsubseteq S$. Then $T \nsubseteq S \cup\{i\}$, and thus $d_{T}^{\delta}(S \cup\{i\})=d_{T}^{\delta}(S)=0$, implying that $d_{T}^{\delta}(S \cup\{i\})=\delta d_{T}^{\delta}(S)$ for any $\delta \in[0,1]$.

(ii) Suppose that $T \subseteq S$. Then $T \subset S \cup\{i\}$, and thus $d_{T}^{\delta}(S)=\delta^{s-t}$ and $d_{T}^{\delta}(S \cup\{i\})=$ $\delta^{s+1-t}$, again implying that $d_{T}^{\delta}(S \cup\{i\})=\delta d_{T}^{\delta}(S)$.

Thus, $i \in N \backslash T$ is a $\delta$-reducing player in $\left(N, d_{T}^{\delta}\right)$. (The remainder of the proof is similar as that in Shapley (1953), but using the basis $d_{T}^{\delta}$ instead of the unanimity basis). ${ }^{14}$ Consider any $(N, v) \in \mathcal{G}$ and $\delta \in[0,1]$. Efficiency and symmetry imply that $\psi_{i}\left(N, d_{N}^{\delta}\right)=\frac{1}{n}$ for all $i \in N$.

Now, let $T \subset N$. Since $i \in N \backslash T$ is a $\delta$-reducing player in $d_{T}^{\delta}$, the $\delta$-reducing player property implies that $\psi_{i}\left(N, d_{T}^{\delta}\right)=0$ for all $i \in N \backslash T$. Efficiency then implies that $\sum_{i \in T} \psi_{i}\left(N, d_{T}^{\delta}\right)=\sum_{i \in N} \psi_{i}\left(N, d_{T}^{\delta}\right)=d_{T}^{\delta}(N)=\delta^{n-t}$. Thus, with symmetry it follows that $\psi_{i}\left(N, d_{T}^{\delta}\right)=\frac{\delta^{n-t}}{t}$ for all $i \in T$. Uniqueness for arbitrary $(N, v) \in \mathcal{G}$ follows since linearity of $\psi$ and the fact that $v=\sum_{\substack{T \subseteq N \\ T \neq \emptyset}} D_{v}^{\delta}(T) d_{T}^{\delta}$ (see Lemma 4.1) imply that $\psi_{i}(N, v)=\sum_{\substack{T \subseteq N \\ T \neq \emptyset}} D_{v}^{\delta}(T) \psi_{i}\left(N, d_{T}^{\delta}\right)=\sum_{\substack{T \subseteq N \\ i \in T}} D_{v}^{\delta}(T) \frac{\delta^{n-t}}{t}$ for all $i \in N$.

We want to stress the difference between the $\delta$-reducing player property and $\delta$ egalitarianism in Joosten (1996) or the quasi-dummy property in Ju et al. (2007). Whereas the latter two properties adapt the null player property by stating what a null player should get, with the $\delta$-reducing player property, similar as in Chameni Nembua (2012), we specify a type of player that gets a zero payoff (like null players according to the null player property and nullifying players according to the nullifying player

\footnotetext{
12 We remark that the results in this section hold if we restrict ourselves to the class of all TU-games on a fixed player set $N$.

13 We remark that uniqueness also can be shown by applying Proposition 2 of Driessen and Radzik (2013) which states that a solution $\psi$ satisfies efficiency, symmetry and linearity if and only if there exists a unique collection of constants $\left\{b_{s} \mid s=1,2, \ldots, n\right\}$ with $b_{n}=1$ such that $\psi_{i}(v)=$ $\sum_{S \subseteq N \backslash\{i\}} \frac{s !(n-s-1) !}{n !}\left(b_{S+1} v(S \cup\{i\})-b_{S}(v(S))\right.$ for all $i \in N$. Take $s \in\{1, \ldots, n\}$ and take any $S \subseteq N$ with $|S|=s$. Then, defining the game $w$ by $w(S)=1, w(S \cup\{i\})=\delta$ and $w(K)=0$ for all $K \in 2^{N} \backslash\{S, S \cup\{i\}$, we see that $i$ is a $\delta$-reducing player in $w$, and thus by the $\delta$-reducing player property $\psi_{i}(w)=0$. Since $\psi_{i}(w)=\frac{s !(n-s-1) !}{n !}\left(b_{s+1} \delta-b_{s}\right)$, it holds that $b_{s+1} \delta=b_{s}$. This holds for all $s \in\{n-1, n-2, \ldots, 1\}$, and thus, by $b_{n}=1$, we get $b_{s}=\delta^{n-s}$ for $s \in\{1,2, \ldots, n\}$. Therefore, $\psi$ is unique and equals the $\delta$-discounted Shapley value. We thank a referee for suggesting this proof.

14 Similar, van den Brink (2007) used the standard basis for characterizing the equal division solution.
} 
property). ${ }^{15}$ Of course, we could also characterize the class of $\delta$-discounted Shapley values $S h^{\delta}$ using a property similar to $\delta$-egalitarianism or the quasi-dummy property by specifying what is earned by null players. It is straightforward to prove that (the obvious proof uses the unanimity basis and is omitted) if $i \in N$ is a null player in $(N, v) \in \mathcal{G}$ then $S h_{i}^{\delta}(N, v)=(1-\delta) \sum_{S \subseteq N \backslash\{i\}} \delta^{n-s-1} \frac{s !(n-s-1) !}{n !} v(S)$.

Since, for monotone games $v(S \cup\{i\}) \geq v(S) \geq \delta v(S)$ for all $\delta \in[0,1]$, we have the following proposition.

Proposition 4.3 Let $0 \leq \delta \leq 1$. If $(N, v) \in \mathcal{G}$ is monotone then $\operatorname{Sh}_{i}^{\delta}(N, v) \geq 0$ for all $i \in N$.

We end this section by mentioning that also other axiomatization of the Shapley value and equal division solution can be modified to characterize the $\delta$-discounted Shapley values. For example, consider the following adaptations of well-known monotonicity axioms. For $\delta \in[0,1]$, solution $\psi$

- satisfies $\delta$-monotonicity if $\psi_{i}(N, v) \geq \psi_{i}(N, w)$ for every pair of games $(N, v),(N, w)$ and $i \in N$ such that $v(S \cup\{i\})-w(S \cup\{i\}) \geq \delta(v(S)-w(S))$ for all $S \subseteq N \backslash\{i\}$.

- satisfies coalitional $\delta$-equivalence if for every pair of games $(N, v),(N, w) \in \mathcal{G}$ it holds that $\psi_{i}(N, v+w)=\psi_{i}(N, v)$ whenever $i$ is a $\delta$-reducing player in $(N, w)$.

Taking $\delta=1$ in $\delta$-monotonicity yields strong monotonicity used to axiomatize the Shapley value by Young (1985), while taking $\delta=1$ in coalitional $\delta$-equivalence yields coalitional strategic equivalence of Chun (1991). Taking $\delta=0$ in these two axioms yields coalitional monotonicity, respectively coalitional standard equivalence, which are used in a similar way to axiomatize the equal division solution in van den Brink (2007). We generalize the results of Young (1985) and Chun (1991). ${ }^{16}$

Theorem 4.4 Let $\delta \in[0,1]$. Then

(i) a solution $\psi$ satisfies efficiency, symmetry and $\delta$-monotonicity if and only if $\psi=S h^{\delta}$.

(ii) a solution $\psi$ satisfies efficiency, symmetry and coalitional $\delta$-equivalence if and only if $\psi=S h^{\delta}$.

Proof It is easy to verify that $S h^{\delta}$ satisfies efficiency, symmetry, $\delta$-monotonicity and coalitional $\delta$-equivalence. Since $\delta$-monotonicity implies coalitional $\delta$-equivalence, for uniqueness we only have to prove uniqueness in (ii). Therefore, suppose that solution $\psi$ satisfies the three axioms of (ii). Now, we show uniqueness by induction on $d(v)=$ $\left|\left\{T \subseteq N \mid D_{v}^{\delta}(T) \neq 0\right\}\right|$ (in a similar way as in Young (1985) and Chun (1991) for the Shapley value and in van den Brink (2007) for the equal division solution).

If $d(v)=0$, then $(N, v)$ is a null game and efficiency and symmetry imply that $\psi_{i}(N, v)=0=\operatorname{Sh}_{i}^{\delta}(N, v)$ for all $i \in N$.

\footnotetext{
15 Instead of using the basis described in Lemma 4.1, we could use Lemma 9 of Ruiz et al. (1998) who give a specific formula that characterizes the class of solutions satisfying efficiency, symmetry and linearity. Because of its relation with the $\delta$-reducing player property we prefer to use the basis $d_{T}^{\delta}, \emptyset \neq T \subseteq N$.

16 Also the axiomatization of Brink (2001) by efficiency, fairness and the null player property can be generalized, but the proof is more tedious and therefore omitted.
} 
Proceeding by induction, assume that $\psi_{i}(N, w)=\operatorname{Sh}_{i}^{\delta}(N, w)$ if $d(w)<d(v)$. Let $H(v)=\left\{i \in N \mid D_{v}^{\delta}(T)=0\right.$ for all $\left.T \subseteq N \backslash\{i\}\right\}$. For every $i \in N \backslash H(v)$ there exists a $T \subseteq N \backslash\{i\}$ such that $D_{v}^{\delta}(T) \neq 0$. Coalitional $\delta$-equivalence and the induction hypothesis imply that $\psi_{i}(N, v)=\psi_{i}\left(N, v-D_{v}^{\delta}(T) d_{T}^{\delta}\right)=S h_{i}^{\delta}\left(N, v-D_{v}^{\delta}(T) d_{T}^{\delta}\right)=$ $S h_{i}^{\delta}(N, v)$ for $i \in N \backslash H(v)$ and $T \subseteq N \backslash\{i\}$. With symmetry and efficiency it then follows that $\psi_{i}(N, v)=\frac{v(N)-\sum_{j \in N \backslash H(v)} S h_{j}^{\delta}(N, v)}{|H(v)|}=S h_{i}^{\delta}(N, v)$ for $i \in H(v)$.

\section{Proof of Theorem 3.1}

In this section we give a more detailed proof of Theorem 3.1. We will use the following two lemma's. First, we provide a recursive formula that generalizes that of Maschler and Owen (1989) for the Shapley value:

$S h_{i}(N, v)=\frac{v(N)-v(N \backslash\{i\})}{n}+\frac{1}{n}\left(\sum_{j \in N \backslash\{i\}} S h_{i}\left(N \backslash\{j\}, v_{N \backslash\{j\}}\right)\right)$ for all $i \in N$

Lemma 5.1 For every $\delta \in[0,1],(N, v) \in \mathcal{G}$ and $i \in N$, it holds that

$$
\operatorname{Sh}_{i}^{\delta}(N, v)=\frac{v(N)-\delta v(N \backslash\{i\})}{n}+\frac{\delta}{n}\left(\sum_{j \in N \backslash\{i\}} S h_{i}^{\delta}\left(N \backslash\{j\}, v_{N \backslash\{j\}}\right)\right)
$$

Proof Take $\delta \in[0,1]$ and $(N, v) \in \mathcal{G}$. If $|N|=1$ then the statement of the theorem is obviously true since $S h_{i}^{\delta}(N, v)=v(\{i\})$ in that case. Proceeding by induction, suppose that the statement is true for all $\left(N^{\prime}, v\right) \in \mathcal{G}$ with $\left|N^{\prime}\right|<|N|$. We already mentioned that it has been shown by Driessen and Radzik (2002) that, for an $n$-player game $(N, v)$, it holds that $\operatorname{Sh}^{\delta}(N, v)=\operatorname{Sh}\left(N, v^{\delta}\right)$ with $\left(N, v^{\delta}\right)$ given by $v^{\delta}(S)=\delta^{n-s} v(S)$, $S \subseteq N$, see (3.1).

But then

$$
\begin{aligned}
S h_{i}^{\delta}(N, v) & =S h_{i}\left(N, v^{\delta}\right) \\
& \left.=\frac{v^{\delta}(N)-v^{\delta}(N \backslash\{i\})}{n}+\frac{1}{n}\left(\sum_{j \in N \backslash\{i\}} S h_{i}(N \backslash\{j\}),\left(v^{\delta}\right)_{N \backslash\{j\}}\right)\right) \\
& \left.=\frac{v(N)-\delta v(N \backslash\{i\})}{n}+\frac{\delta}{n}\left(\sum_{j \in N \backslash\{i\}} S h_{i}(N \backslash\{j\}),\left(v_{N \backslash\{j\}}\right)^{\delta}\right)\right) \\
& =\frac{v(N)-\delta v(N \backslash\{i\})}{n}+\frac{\delta}{n}\left(\sum_{j \in N \backslash\{i\}} S h_{i}^{\delta}\left(N \backslash\{j\}, v_{N \backslash\{j\}}\right)\right),
\end{aligned}
$$


where the first equality follows from (3.1), the second equality follows from (5.1) and the definition of $v^{\delta}$, the third equality follows from ${ }^{17}$ the fact that $\left(v^{\delta}\right)_{N \backslash\{j\}}=$ $\delta\left(v_{N \backslash\{j\}}\right)^{\delta}$, and the fourth equality again follows from (3.1).

Second, in proving Theorem 3.1 we also use a modified balanced contributions property. Recall that Myerson (1980)'s balanced contributions property of the Shapley value states that

$$
S h_{i}(N, v)-S h_{i}\left(N \backslash\{j\}, v_{N \backslash\{j\}}\right)=S h_{j}(N, v)-S h_{j}\left(N \backslash\{i\}, v_{N \backslash\{i\}}\right) .
$$

Lemma 5.2 For any game $(N, v)$, and for all $i, j \in N$ with $i \neq j$,

$$
\operatorname{Sh}_{i}^{\delta}(N, v)-\delta \operatorname{Sh}_{i}^{\delta}\left(N \backslash\{j\}, v_{N \backslash\{j\}}\right)=\operatorname{Sh}_{j}^{\delta}(N, v)-\delta \operatorname{Sh}_{j}^{\delta}\left(N \backslash\{i\}, v_{N \backslash\{i\}}\right)
$$

Proof The proof follows straightforward since (3.1), (5.2) and footnote 17 imply that $\operatorname{Sh}_{i}^{\delta}(N, v)-\delta S h_{i}^{\delta}\left(N \backslash\{j\}, v_{N \backslash\{j\}}\right)=\operatorname{Sh}_{i}\left(N, v^{\delta}\right)-\delta S h_{i}\left(N \backslash\{j\},\left(v_{N \backslash\{j\}}\right)^{\delta}\right)=$ $S h_{i}\left(N, v^{\delta}\right)-\delta S_{i}\left(N \backslash\{j\}, \frac{1}{\delta}\left(v^{\delta}\right)_{N \backslash\{j\}}\right)=\operatorname{Sh}_{i}\left(N, v^{\delta}\right)-\operatorname{Sh}_{i}\left(N \backslash\{j\},\left(v^{\delta}\right)_{N \backslash\{j\}}\right)=$ $S h_{j}\left(N, v^{\delta}\right)-S h_{j}\left(N \backslash\{i\},\left(v^{\delta}\right)_{N \backslash\{i\}}\right)=S h_{j}\left(N, v^{\delta}\right)-\delta S h_{j}\left(N \backslash\{i\}, \frac{1}{\delta}\left(v^{\delta}\right)_{N \backslash\{i\}}\right)=$ $S h_{j}\left(N, v^{\delta}\right)-\delta S h_{j}\left(N \backslash\{i\},\left(v_{N \backslash\{i\}}\right)^{\delta}\right)=S h_{j}^{\delta}(N, v)-\delta S h_{j}^{\delta}\left(N \backslash\{i\}, v_{N \backslash\{i\}}\right)$, where the first equality follows from (3.1), the second equality follows from footnote 17 , the fourth equality follows from (5.2), and the following equalities follow similar as the previous ones with the roles of players $i$ and $j$ reversed.

Next we show how the proof of the implementation of the Shapley value in PérezCastrillo and Wettstein (2001), can be modified to prove Theorem 3.1.

Proof of Theorem 3.1 The proof follows the lines of the proof of the implementation of the Shapley value in Pérez-Castrillo and Wettstein (2001, Theorem 1), and therefore we only mention the differences. ${ }^{18}$ First, Pérez-Castrillo and Wettstein (2001) construct a strategy profile that is a subgame perfect equilibrium (SPE) in their bidding game and yields the Shapley value payoffs as outcomes. In this strategy profile, in all actions of player $i$ we discount the part that is based on payoffs in restricted games on the set of players without the proposer $\alpha_{t}$ (in the bidding in stage 1, the proposals in stage 2 if $i$ is the proposer, and what proposals $i$ will accept in stage 3 if $i$ is not the proposer), i.e. in round $t \in\{1, \ldots, n-1\}$ we just replace payoffs $\phi_{j}\left(N_{t} \backslash\{i\}\right)$ by $\delta \phi_{j}\left(N_{t} \backslash\{i\}\right)$ and replace $\phi_{i}\left(N_{t} \backslash\{j\}\right)$ by $\delta \phi_{i}\left(N_{t} \backslash\{j\}\right) .{ }^{19}$ All net bids being zero can be shown using the modified balanced contributions (5.3) instead of balanced contributions.

Checking that the corresponding strategies yield an SPE goes similar as in PérezCastrillo and Wettstein (2001), but under the condition of $\delta$-monotonicity.

\footnotetext{
17 This can be seen since for all $S \subseteq N \backslash\{j\}$ we have $\left(v_{N \backslash\{j\}}\right)^{\delta}(S)=\delta^{n-1-s} v(S)=\delta^{-1} \delta^{n-s} v(S)=$ $\delta^{-1}\left(v^{\delta}\right)_{N \backslash\{j\}}(S)$.

18 The full proof can be obtained from the authors on request.

19 Pérez-Castrillo and Wettstein (2001) shorten payoffs $\phi_{i}\left(T, v_{T}\right)$ by $\phi_{i}(T)$ for $T \subset N$ which we will also do in this proof. They use the notation $N$ for the player set in any subgame, while in our notation $N$ refers to the full player set, and subgames are played on subsets $N_{t}$ in round $t \in\{1, \ldots, n-1\}$.
} 
Then, Pérez-Castrillo and Wettstein (2001) show that any SPE yields the Shapley value payoffs by a series of claims. Claim (a) states that in any SPE all players $i \neq \alpha_{t}$ that are not proposer in round $t \in\{1, \ldots, n-1\}$ accept the offer of proposer $\alpha_{t}$ if that is greater than their payoff $\phi_{i}\left(N_{t} \backslash\left\{\alpha_{t}\right\}\right)$, and the offer is rejected if at least one of those players $i \neq \alpha_{t}$ gets an offer smaller than $\phi_{i}\left(N_{t} \backslash\left\{\alpha_{t}\right\}\right)$. We just discount the payoffs on the player set $N_{t} \backslash\left\{\alpha_{t}\right\}$ by multiplying these payoffs by $\delta$, i.e. replacing $\phi_{i}\left(N_{t} \backslash\left\{\alpha_{t}\right\}\right)$ by $\delta \phi_{i}\left(N_{t} \backslash\left\{\alpha_{t}\right\}\right)$.

Claim (b) states that if $v\left(N_{t}\right)>v\left(N_{t} \backslash\left\{\alpha_{t}\right\}\right)+v\left(\left\{\alpha_{t}\right\}\right)$, the only SPE of the (sub)game that starts at stage 2 is such that the proposer offers $\phi_{i}\left(N_{t} \backslash\left\{\alpha_{t}\right\}\right)$ to all $i \neq \alpha_{t}$, and at stage 3 every player $i \neq \alpha_{t}$ accepts any offer greater or equal than $\phi_{i}\left(N_{t} \backslash\left\{\alpha_{t}\right\}\right)$ and rejects all smaller offers. Again we simply discount the payoffs of restricted games and replace $\phi_{i}\left(N_{t} \backslash\left\{\alpha_{t}\right\}\right)$ by $\delta \phi_{i}\left(N_{t} \backslash\left\{\alpha_{t}\right\}\right)$. Using $\delta$-monotonicity, this claim is shown if $v\left(N_{t}\right)>\delta v\left(N_{t} \backslash\left\{\alpha_{t}\right\}\right)+v\left(\left\{\alpha_{t}\right\}\right)$, i.e. we also discount the surplus $v\left(N_{t} \backslash\left\{\alpha_{t}\right\}\right)$. (Also in the proof of this claim everywhere we replace $v\left(N_{t} \backslash\left\{\alpha_{t}\right\}\right)$ by $\delta v\left(N_{t} \backslash\left\{\alpha_{t}\right\}\right.$ ).

Claim (c) of Pérez-Castrillo and Wettstein (2001) states that in any SPE, the net bids of all players are equal, and therefore equal to zero. For this claim discounting is not relevant, and for our mechanism this is proved in the same way. ${ }^{20}$

Claim (d) of Pérez-Castrillo and Wettstein (2001) states that in any SPE, each player's payoff is the same regardless who is chosen as the proposer. Also the proof of this claim does not need discounting, and is identical for all discounted Shapley values.

Finally Claim (e), stating that in any SPE the final payoff received by each of the players coincides with their Shapley value, uses Maschler and Owen (1989)'s recursive formula for the Shapley value [see (5.1)]. Discounting the payoff $v\left(N_{t} \backslash\{i\}\right)$ by $\delta v\left(N_{t} \backslash\{i\}\right)$, we can apply Lemma 5.1 to obtain a similar result for any discounted Shapley value.

This completes the proof.

\section{Concluding remarks}

In this paper we provided a strategic implementation and axiomatic characterization of the discounted Shapley values. First, we gave a strategic implementation by modifying the bidding mechanism of Pérez-Castrillo and Wettstein (2001) implementing the Shapley value, but allowing discounting of the worths to be allocated after every rejection of the proposal.

Second, we provided an axiomatization of the $\delta$-discounted Shapley values by replacing the null-, respectively, nullifying player property that are used in characterizations of the Shapley value, respectively, the equal division solution, by the $\delta$-reducing player property (with the null- and nullifying player properties as special cases). ${ }^{21}$

\footnotetext{
20 In the proof of this claim in Pérez-Castrillo and Wettstein (2001) the player $j \notin \Omega$ should be one with the highest net bid among those not in $\Omega$, where $\Omega$ is the set of players in $N$ with the highest net bid.

21 Although among the efficient symmetric solutions the Shapley value is the most marginalistic solution (see Young 1985), there are different possibilities with respect to the most egalitarian solution. An alternative to the equal division solution is the CIS-value, or equal surplus division solution, which assigns to every TU-game the Center of the Imputation Set, i.e. every player gets its singleton worth and the remaining
} 
Table 1 Discounting and consistency

\begin{tabular}{lll}
\hline & $\begin{array}{l}\text { Discounting in } \\
\text { implementation }\end{array}$ & $\begin{array}{l}\text { Reduced game } \\
\text { consistency }\end{array}$ \\
\hline $\begin{array}{l}\text { Discounted Shapley values } \\
\text { Egalitarian Shapley values }\end{array}$ & $\begin{array}{l}\text { Every round } \\
\text { Only in first round }\end{array}$ & $\begin{array}{l}\text { Hart and Mas-Colell } \\
\text { Sobolev }\end{array}$ \\
\hline
\end{tabular}

We already mentioned that van den Brink et al (2013) showed that the egalitarian Shapley values (i.e. convex combinations of the Shapley value and equal division solution) of Joosten (1996) can be implemented by allowing the possibility of breakdown to occur only in the first round, while the discounted Shapley values are implemented by allowing this possibility of breakdown (or discounting) in every round. It is a plan for future research to consider what classes of solutions are obtained by a strategic implementation where discounting is applied only in the first $k \in\{2, \ldots n-1\}$ rounds. Moreover, we might consider different discount factors in different rounds, and for different players.

Whereas Joosten (1996) showed that all discounted Shapley values satisfy Hart and Mas-Colell consistency, van den Brink et al. (2013) showed that all egalitarian Shapley values satisfy Sobolev's reduced game consistency (Sobolev 1973).

This gives another comparison between discounted and egalitarian Shapley values (we summarize these comparisons in Table 1), and the natural question what kind of consistency properties are satisfied by solutions obtained from the strategic implementation where discounting is applied only in the first $k \in\{2, \ldots n-1\}$ rounds.

Acknowledgments Financial supports from NWO-Grant B 45-299 and JSPS KAKENHI Grant Number 26380247 are gratefully acknowledged. We thank anonymous referees for their valuable comments.

Open Access This article is distributed under the terms of the Creative Commons Attribution 4.0 International License (http://creativecommons.org/licenses/by/4.0/), which permits unrestricted use, distribution, and reproduction in any medium, provided you give appropriate credit to the original author(s) and the source, provide a link to the Creative Commons license, and indicate if changes were made.

\section{References}

Chun Y (1991) On the symmetric and weighted Shapley values. Int J Game Theory 20:183-190

Deegan J, Packel EW (1979) A new index of power for simple n-person games. Int J Game Theory 7:113-123

Driessen TSH, Funaki Y (1991) Coincidence of and collinearity between game theoretic solutions. OR Spektrum 13:15-30

Driessen TSH, Radzik T (2002) A weighted pseudo-potential approach to values for TU games. Int Trans Oper Res (ITOR) 9:1-18

Driessen TSH, Radzik T (2013) On a family of values for TU-games generalizing the Shapley value. Math Soc Sci 65:105-111

Gul F (1989) Bargaining foundations of Shapely value. Econometrica 57:81-95

Footnote 21 continued

surplus is distributed equally among the players, see Driessen and Funaki (1991). Different egalitarian solutions are compared in van den Brink and Funaki (2009). 
Harsanyi JC (1959) A Bargaining Model for Cooperative N-person Games. In: Tucker AW, Luce RD (eds) Contributions to the Theory of Games IV. Princeton, Princeton UP, pp 325-355

Hart S, Mas-Colell A (1988) The Potential of the Shapley Value. In: Roth AE (ed) The Shapley Value. Essays in Honor of L.S. Shapley. Cambridge University Press, Cambridge, pp 127-137

Hart S, Mas-Colell A (1989) Potential, value and consistency. Econometrica 57:589-614

Hart S, Mas-Colell A (1996) Bargaining and Value. Econometrica 64:357-380

Joosten R (1996) Dynamics, equilibria and values, dissertation. Maastricht University, The Netherlands

Ju Y, Borm PEM, Ruys PHM (2007) The consensus value : a new solution concept for cooperative games. Soc Choice Welf 28:685-703

Ju Y, Wettstein D (2009) Implementing cooperative solution concepts: a generalized bidding approach. Econ Theory 39:307-330

Malawski M (2013) Procedural values for cooperative games. Int J Game Theory 42:305-324

Maschler M, Owen G (1989) The consistent Shapley value for Hyperplane games. Int J Game Theory 18:389-407

Myerson RB (1980) Conference structures and fair allocation rules. Int J Game Theory 9:169-182

Nash JF (1953) Two-person cooperative games. Econometrica 21:128-140

Nembua CC (2012) Linear efficient and symmetric values for TU-Games: sharing the joint gain of cooperation. Games Econ Behav 74:431-433

Pérez-Castrillo D, Wettstein D (2001) Bidding for the surplus: a non-cooperative approach to the Shapley value. J Econ Theory 100:274-294

Rubinstein A (1982) Perfect equilibrium in a bargaining model. Econometrica 50:97-109

Ruiz LM, Valenciano F, Zarzuelo JM (1998) The family of least square values for transferable utility games. Games Econ Behav 24:109-130

Shapley LS (1953) A Value for $n$-Person games. In: Kuhn HW, Tucker AW (eds) Annals of Mathematics Studies 28 (Contributions to the Theory of Games, Vol. 2). Princeton University Press, Princeton, pp 307-317

Sobolev AI (1973) "The Functional Equations that Give the Payoffs of the Players in an N-Person Game", in: Advaces in Game Theory (ed. E. Vilkas), Izdat. "Mintis", Vilnius, pp. 151-153 (in Russion)

van den Brink R (2001) An axiomatization of the Shapley value using a fairness property. Int J Game Theory 30:309-319

van den Brink R (2007) Null or nullifying players: the difference between the Shapley value and equal division solutions. J Econ Theory 136:767-775

van den Brink R, Funaki Y (2009) Axiomatizations of a class of equal Surplus sharing solutions for TUGames. Theory Decis 67:303-340

van den Brink R, Funaki Y, Ju Y (2013) Reconciling marginalism with egalitarianism: consistency monotonicity, and implementation of egalitarian Shapley values. Soc Choice Welf 40:693-714

Young HP (1985) Monotonic solutions of cooperative games. Int J Game Theory 14:65-72 